\title{
Thermally activated escape rates of uniaxial spin systems with transverse field: Uniaxial crossovers
}

\author{
D. A. Garanin* \\ Max-Planck-Institut für Physik komplexer Systeme, Nöthnitzer Strasse 38, D-01187 Dresden, Germany \\ E. C. Kennedy ${ }^{\dagger}$ and D. S. F. Crothers \\ Department of Applied Mathematics and Theoretical Physics, The Queen's University of Belfast, Belfast, BT7 1 NN, Northern Ireland \\ W. T. Coffey \\ Department of Electronic and Electrical Engineering, Trinity College, Dublin 2, Ireland
}

(Received 11 March 1999)

\begin{abstract}
Classical escape rates of uniaxial spin systems are characterized by a prefactor differing from and much smaller than that of the particle problem, since the maximum of the spin energy is attained everywhere on the line of constant latitude: $\theta=$ const, $0 \leqslant \varphi \leqslant 2 \pi$. If a transverse field is applied, a saddle point of the energy is formed, and high, moderate, and low damping regimes (similar to those for particles) appear. Here we present the first analytical and numerical study of crossovers between the uniaxial and other regimes for spin systems. It is shown that there is one HD-Uniaxial crossover, whereas at low damping the uniaxial and LD regimes are separated by two crossovers. [S1063-651X(99)10512-9]
\end{abstract}

PACS number(s): 05.40.- a, 75.50.Tt

The study of thermal activation escape rates of fine magnetic particles, which are usually modelled as classical spins with predominantly uniaxial anisotropy, may be traced from the early predictions of Néel [1] through the first theoretical treatments of Brown [2,3] to the recent experiments of Wernsdorfer et al. [4] on individual magnetic particles of controlled form. These experiments allow one to check the Stoner-Wohlfarth angular dependence of the switching field [5] and to make a comparison [6,7] with existing theories where the energy barrier is reduced by applying a magnetic field. The theories checked are those for the intermediate-tohigh damping (IHD) case $[3,8,9]$, as well as for the lowdamping (LD) case [10].

The IHD and LD limits for spins are similar to those for the particle problem, which were established by Kramers [11]. The most significant difference is that for spins in the HD limit the prefactor $\Gamma_{0}$ in the escape rate $\Gamma=\Gamma_{0}$ exp $(-\Delta U / T)$ behaves as $\Gamma_{0} \propto a$, where $a$ is the damping constant [if the Landau-Lifshitz (LL) equation is used], whereas for particles $\Gamma_{0} \propto 1 / a$. A question which has not yet been addressed, both theoretically and experimentally, and which is the subject of this paper, is how these three dampinggoverned regimes merge into the single uniaxial regime [2] if the field is removed?

Let us consider the Hamiltonian

$$
\mathcal{H}=-\widetilde{K} n_{z}^{2}-\mu_{0} \mathbf{n} \cdot \mathbf{H}, \quad|\mathbf{n}|=1,
$$

where $\mu_{0}=M_{s} V$ is the magnetic moment and $\widetilde{K}=K V$ is the uniaxial anisotropy energy of the particle. The Fokker-

\footnotetext{
*Electronic address: www.mpipks-dresden.mpg.de/ garanin/, garanin@mpipks-dresden.mpg.de

${ }^{\dagger}$ Electronic address: e.kennedy@hornet.am.qub.ac.uk

${ }^{\ddagger}$ Electronic address: secr@mee.tcd.ie
}

Planck equation for the distribution function of the spins $f(\theta, \varphi, t)$, which follows from the stochastic LL equation, reads

$$
\frac{\partial f}{\partial t}+\frac{\partial j_{x}}{\partial x}+\frac{\partial j_{\varphi}}{\partial \varphi}=0
$$

where $x \equiv \cos \theta$,

$$
\begin{gathered}
j_{x}=\frac{\gamma}{\mu_{0}}\left[-\frac{\partial \mathcal{H}}{\partial \varphi} f-a\left(1-x^{2}\right)\left(\frac{\partial \mathcal{H}}{\partial x}+T \frac{\partial}{\partial x}\right) f\right], \\
j_{\varphi}=\frac{\gamma}{\mu_{0}}\left[\frac{\partial \mathcal{H}}{\partial x} f-\frac{a}{1-x^{2}}\left(\frac{\partial \mathcal{H}}{\partial \varphi}+T \frac{\partial}{\partial \varphi}\right) f\right] .
\end{gathered}
$$

To solve the FPE at low temperatures, $T \ll \Delta U$, we represent $f$ as $f(\mathbf{N}, t)=f_{\text {eq }}(\mathbf{N}) g(\mathbf{N}, t)$, where $f_{\text {eq }}(\mathbf{N})=\mathcal{Z}^{-1}$ exp $[-\mathcal{H}(\mathbf{N}) / T]$ is the equilibrium distribution function. Neglecting the exponentially small $\dot{g}$ one obtains

$$
\begin{gathered}
\frac{\partial \mathcal{H}}{\partial \varphi} \frac{\partial g}{\partial x}-\frac{\partial \mathcal{H}}{\partial x} \frac{\partial g}{\partial \varphi}+a\left[\left(-\frac{\partial \mathcal{H}}{\partial x}+T \frac{\partial}{\partial x}\right)\left(1-x^{2}\right) \frac{\partial g}{\partial x}\right. \\
\left.+\frac{1}{1-x^{2}}\left(-\frac{\partial \mathcal{H}}{\partial \varphi}+T \frac{\partial}{\partial \varphi}\right) \frac{\partial g}{\partial \varphi}\right]=0 .
\end{gathered}
$$

The function $g$ assumes the values $g_{1}$ and $g_{2}$ in the wells and changes in a narrow region about the top of the barrier, so that

$$
g(x, \varphi)=g_{1}+\left(g_{2}-g_{1}\right) \zeta(x, \varphi),
$$

where $\zeta$ assumes the values 0 and 1 in the first and second wells, respectively. The numbers of particles in the wells 
satisfy $N_{1}+N_{2}=1$ and $N_{i}=g_{i} N_{i \text {, eq }}$, where $N_{i \text {, eq }}=\mathcal{Z}_{i} / \mathcal{Z}$ are the equilibrium values and $\mathcal{Z}_{1}$ and $\mathcal{Z}_{2}$ are partition functions for each of the wells.

The change in the particle number in the first well $N_{1}$ is due to the flow of particles from the first to the second well through the line $x=$ const (say, the equator $x=0$ ): $\dot{N}_{1}$ $=-\int_{0}^{2 \pi} d \varphi j_{x}$. Using $f_{\text {eq }} \partial \mathcal{H} / \partial \varphi=-T \partial f_{\text {eq }} / \partial \varphi$, one can integrate the first term of $j_{x}$ by parts. Finally, using Eq. (5), one has the kinetic equations

$$
\dot{N}_{1}=-\dot{N}_{2}=\Gamma\left(N_{2} N_{1, \text { eq }}-N_{1} N_{2, \text { eq }}\right),
$$

the escape rate being

$$
\Gamma=\left[\frac{1}{\mathcal{Z}_{1}}+\frac{1}{\mathcal{Z}_{2}}\right] \frac{\gamma T}{\mu_{0}} \int_{0}^{2 \pi} d \varphi e^{-\mathcal{H} / T}\left[a\left(1-x^{2}\right) \frac{\partial \zeta}{\partial x}+\frac{\partial \zeta}{\partial \varphi}\right],
$$

where $\zeta$ satisfies Eq. (4) with $g \Rightarrow \zeta$ and with the boundary conditions stated in after Eq. (5). Also, one can calculate a current through a line of constant energy surrounding one of the wells, which is appropriate in the LD case.

For the transverse-field (unbiased) model

$$
\mathcal{H}(x, \varphi) / T \equiv \varepsilon=-\sigma\left(x^{2}+2 h \sqrt{1-x^{2}} \cos \varphi\right),
$$

where

$$
\sigma \equiv \frac{\widetilde{K}}{T}=\frac{K V}{T}, \quad \mathbf{h} \equiv \frac{\mu_{0} \mathbf{H}}{2 \widetilde{K}}=\frac{\mathbf{H} M_{s}}{2 K} .
$$

Another useful dimensionless variable is $\xi \equiv 2 \sigma h$ $=H M_{s} V / T$. The function $\zeta$ satisfies

$$
\begin{aligned}
(1- & h \cos \varphi) x\left(a \frac{\partial \zeta}{\partial x}+\frac{\partial \zeta}{\partial \varphi}\right)+h \sin \varphi\left(\frac{\partial \zeta}{\partial x}-a \frac{\partial \zeta}{\partial \varphi}\right) \\
+ & \frac{a}{2 \sigma}\left(\frac{\partial^{2} \zeta}{\partial x^{2}}+\frac{\partial^{2} \zeta}{\partial \varphi^{2}}\right)=0,
\end{aligned}
$$

where $1-x^{2} \rightarrow 1$ in the region $x \ll 1$, which is relevant in the uniaxial and IHD cases. The result for $\Gamma$ can be written

$$
\Gamma=\frac{\omega_{1}}{\pi} A \exp \left[-\frac{\Delta U}{T}\right], \quad \omega_{1}=\frac{2 \gamma K}{M_{s}} \sqrt{1-h^{2}},
$$

where $\Delta U=\mathcal{H}_{\mathrm{sad}}-\mathcal{H}_{\text {min }}, \Delta U / T=\sigma(1-h)^{2}$, and $\omega_{1}$ is the ferromagnetic resonance frequency near the bottom of the well (the attempt frequency), the factor $A$ describes deviations from the transition-state theory (TST), and the lack of the factor 2 in the denominator is due to the two symmetric wells.

In the uniaxial case, $h=0$, the function $\zeta$ is independent of $\varphi$, and the solution of Eq. (10) is $\partial \zeta / \partial x=\sqrt{\sigma / \pi} \exp$ $\left(-\sigma x^{2}\right)$. Equation (7) then yields [2]

$$
A=2 \pi a \sqrt{\sigma / \pi} .
$$

Another analytically solvable case is IHD with a pronounced saddle: $T \ll \mathcal{H}_{\text {max }}-\mathcal{H}_{\text {sad }}$, i.e., $\xi \equiv 2 \sigma h \gg 1$ for our model. Here the coefficients in Eq. (10) for $\zeta$ can be linearized near the saddle point $x=\varphi=0$ shown in Fig. 1. Next one seeks a solution $\zeta(x, \varphi)=\zeta(u)$ with $u=x+\nu \varphi$ and ap-

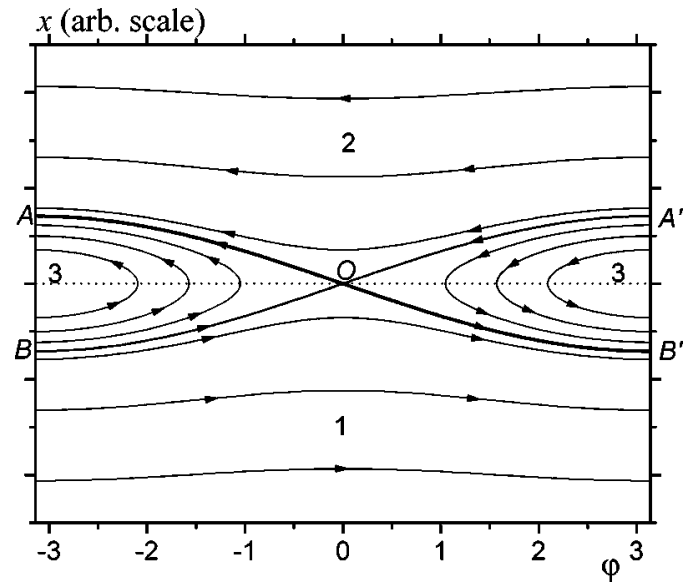

FIG. 1. Trajectories of a magnetic particle with uniaxial anisotropy and transverse field. Regions 1 and 2 are the potential wells, $O$ is the saddle point, and 3 is the maximum of the potential.

propriately chosen $\nu[3,11]$. This form of $\zeta(x, \varphi)$ implies that it changes for $\sigma \gg 1$ in a narrow region across the line $A O B^{\prime}$ in Fig. 1. Evaluating $\partial \zeta(u) / \partial u$, from Eq. (7) one obtains [9]

$$
A=\frac{a(1-2 h)+\sqrt{a^{2}+4 h(1-h)}}{2 \sqrt{h(1-h)}}
$$

in Eq. (11), with the limiting forms

$$
A \cong \begin{cases}1, & a^{2} \ll h(1-h) \\ \sqrt{1-h}\left(1+a^{2}\right) / a, & 1-h \ll 1, a^{2} \\ a / \sqrt{h}, & h \ll 1, a^{2} .\end{cases}
$$

Note that for $h \ll 1$ the HD regime is attained already for $a$ $\gtrsim \sqrt{h} \ll 1$, where the difference between the LL and Gilbert equations is still irrelevant. For $h<1 / 2$, Eq. (13) monotonically increases with $a$. For $h>1 / 2$, it has a minimum at $a$ $=2 h-1$. In the limit $h \rightarrow 0$ the result for $A$ and thus the escape rate $\Gamma$ diverges, because the saddle becomes infinitely wide. This divergence is, however, unphysical, because Eq. (13) requires $\xi \equiv 2 h \sigma \gg 1$.

For small $a$, the IHD formula above fails, because the tacit assumption that the magnetic moments approaching the barrier from the depth of the well are in thermal equilibrium is violated. In the LD limit, the situation becomes completely different: The function $g$ changes in a narrow region along the lines corresponding to the saddle-point energy $\varepsilon_{c}$, i.e., across the lines $A O A^{\prime}$ and $B O B^{\prime}$ in Fig. 1. Diffusion in the energy space becomes very slow, and thus $g$ equilibrates along the lines $\varepsilon=$ const. That is, $\zeta$ of Eq. (5) can be approximated as

$$
\zeta(x, \varphi) \cong \zeta(\varepsilon), \quad \varepsilon=\varepsilon(x, \varphi) \equiv \mathcal{H}(x, \varphi) / T
$$

Then the conservative part of Eq. (4) for $\zeta$ vanishes. The rest can be averaged over the phase variable, i.e., over the constant-energy line, and written

$$
\frac{d^{2} \zeta}{d \varepsilon^{2}}=\left[1-\frac{A(\varepsilon)}{B(\varepsilon)}\right] \frac{d \zeta}{d \varepsilon},
$$


where

$$
\begin{gathered}
A(\varepsilon) \equiv\left\langle\frac{\partial}{\partial x}\left(1-x^{2}\right) \frac{\partial \varepsilon}{\partial x}+\frac{1}{1-x^{2}} \frac{\partial^{2} \varepsilon}{\partial \varphi^{2}}\right\rangle, \\
B(\varepsilon) \equiv\left\langle\left(1-x^{2}\right)\left(\frac{\partial \varepsilon}{\partial x}\right)^{2}+\frac{1}{1-x^{2}}\left(\frac{\partial \varepsilon}{\partial \varphi}\right)^{2}\right\rangle,
\end{gathered}
$$

with the averaging over conservative trajectories.

Usually $A / B \sim T / E_{\text {char }}$, with $E_{\text {char }}$ a characteristic energy, can be neglected at low temperatures, and Eq. (16) is easily integrated. Calculating the flux across the part of the separatrix $\varepsilon=\varepsilon_{c}$ around the first well yields [10]

$$
A=\frac{a}{2} \oint_{\varepsilon=\varepsilon_{c}}\left[\left(1-x^{2}\right) \frac{\partial \varepsilon}{\partial x} d \varphi-\frac{1}{1-x^{2}} \frac{\partial \varepsilon}{\partial \varphi} d x\right]
$$

i.e., $A=\widetilde{\delta} / 2$, where $\widetilde{\delta}=\delta / T$ and $\delta$ is the energy dissipated over the period of the precession at the saddle-point energy in the low-damping case, coinciding with the result for particles in the LD limit [11], the factor $1 / 2$ arising because of the two symmetric wells.

If the transverse field satisfies $h \ll 1$, then Eq. (18) greatly simplifies. One can set $1-x^{2} \Rightarrow 1$ everywhere and retain only the leading term containing $\partial \varepsilon / \partial x \cong-2 \sigma x$, where along the separatrix $\quad x=x(\varphi) \cong-\sqrt{2 h(1-\cos \varphi)}=-2 \sqrt{h} \sin (\varphi / 2)$. This results in

$$
A \cong a \sigma \int_{0}^{2 \pi} x(\varphi) d \varphi=8 a \sigma \sqrt{h}
$$

vanishing in the limit $h \rightarrow 0$, instead of approaching Eq. (12), and becoming invalid at $\xi \lesssim 1$ (see below).

Let us now study crossovers to the uniaxial regime from the HD and LD regimes, starting from the HD one. For large values of $\sigma$ one expects a crossover to the uniaxial case for $h \sim 1 / \sigma$, which is so small that the function $\zeta$ does not yet deviate from its uniaxial form written in the line above Eq. (12). Under this assumption which will be checked shortly, the only effect of the transverse field is that in Eq. (7) $f_{\text {eq }}$ $\propto \exp (\xi \cos \varphi)$. Integration over $\varphi$ yields

$$
A=2 \pi a \sqrt{\sigma / \pi} \exp (-\xi) I_{0}(\xi)
$$

which interpolates between Eq. (12) and the third line of Eq. (14), so describing a crossover between the uniaxial and HD regimes. If $a \ll 1$, then at higher fields, $h \gtrsim a^{2}$, a crossover to the ID regime with $A=1$ occurs, which is described by Eq. (13). Thus Eq. (20) applies if $1 / \sigma \ll a^{2}$, i.e., $\alpha \equiv a \sqrt{\sigma} \gg 1$.

Let us now consider LD for $h \ll 1$ more accurately. Here $A$ and $B$ of Eq. (17) simplify to $A(\varepsilon) \cong\left\langle\partial^{2} \varepsilon / \partial x^{2}\right\rangle \cong-2 \sigma$ and $B(\varepsilon) \cong\left\langle(\partial \varepsilon / \partial x)^{2}\right\rangle \cong-4 \sigma \varepsilon$, so that Eq. (16) becomes

$$
\frac{d^{2} \zeta}{d \varepsilon^{2}}=\left[1-\frac{1}{2 \varepsilon}\right] \frac{d \zeta}{d \varepsilon} \Rightarrow \frac{d \zeta}{d \varepsilon}=\frac{C}{\sqrt{-\varepsilon}} e^{\varepsilon-\varepsilon_{c}},
$$

where the constant $C$ is determined by the condition

$$
2 C \int_{-\infty}^{\varepsilon_{c}} \frac{d \varepsilon}{\sqrt{-\varepsilon}} \exp \left(\varepsilon-\varepsilon_{c}\right)=1, \quad \varepsilon_{c}=-\xi .
$$

Note that for $\xi \lesssim 1$ the term $1 /(2 \varepsilon)$ in Eq. (21) cannot be dropped, since $-\varepsilon \sim-\varepsilon_{c}=\xi$. This is the difference from the standard LD case above.

Thus, instead of Eq. (19),

$$
A \cong a C \lim _{\varepsilon \rightarrow \varepsilon_{c}=-\xi} \frac{\oint(\partial \varepsilon / \partial x) d \varphi}{\sqrt{-\varepsilon}}=2 \pi a \sqrt{\sigma / \pi} f(\xi) Q,
$$

where

$$
Q=\lim _{\varepsilon \rightarrow-\xi} \frac{1}{2 \pi} \int_{0}^{2 \pi} d \varphi \sqrt{\frac{-\varepsilon-\xi \cos \varphi}{-\varepsilon}}
$$

and

$$
f(\xi)=\sqrt{\pi}\left(\int_{0}^{\infty} \frac{d t e^{-t}}{\sqrt{\xi+t}}\right)^{-1}=\left(e^{\xi} \operatorname{erfc} \sqrt{\xi}\right)^{-1}
$$

One has $Q=1$ for $\xi=0$ and $Q=2^{3 / 2} / \pi \approx 0.900$ for $\xi \neq 0$, whereas the asymptotes of the function $f(\xi)$ are

$$
f(\xi) \cong \begin{cases}1+2 \sqrt{\xi / \pi}, & \xi \ll 1 \\ \sqrt{\pi \xi}, & \xi \gg 1 .\end{cases}
$$

Thus for $\xi=0$ the uniaxial limit, Eq. (12), is recovered. In the region $\xi \sim 1$, the function $f(\xi)$ describes the crossover to the standard LD result of Eq. (19). The discontinuous form of $Q$ above shows that our treatment was not accurate enough to describe yet another crossover at $\xi \sim \sqrt{\alpha}$, where $\alpha \equiv a \sqrt{\sigma}$ is small in the LD case (see below).

It follows that the parameters governing the uniaxial crossover are

$$
\xi=2 h \sigma=H M_{s} V / T, \quad \alpha=a \sqrt{\sigma} .
$$

For $\sigma \gg 1$, in the relevant region $\xi \sim 1$ one has $h \ll 1$. Thus in Eq. (10) the terms $h \cos \varphi, a \partial \zeta / \partial \varphi$, and $\partial^{2} \zeta / \partial \varphi^{2}$ can be neglected, the derivatives with respect to $\varphi$ being much smaller than those with respect to $x$. In contrast, the terms $h \sin \varphi \partial \zeta / \partial x$ and $\partial \zeta / \partial \varphi$ become relevant for small $a$ and should be retained. The resulting equation can be cast into the scaled form

$$
\alpha\left(\frac{\partial^{2} \zeta}{\partial \tilde{x}^{2}}+2 \tilde{x} \frac{\partial \zeta}{\partial \tilde{x}}\right)+2 \tilde{x} \frac{\partial \zeta}{\partial \varphi}+\xi \sin \varphi \frac{\partial \zeta}{\partial \tilde{x}}=0
$$

where $\tilde{x} \equiv \sqrt{\sigma} x$ and the boundary conditions are $\zeta=0$ for $\tilde{x}$ $=-\infty$ and $\zeta=1$ for $\tilde{x}=\infty$.

The "phase diagram" of the regimes for the escape rate of the uniaxial model with transverse field is shown in Fig. 2. The ID-HD crossover is described by Eq. (13) and occurs at $a^{2} \sim h$, i.e., $\alpha \sim \sqrt{\xi}$. The ID-LD crossover occurs if in Eq. (19) $A \sim 1$, which amounts to $\alpha \sim 1 / \sqrt{\xi}$. Crossover of this type for the particle problem is described by Melnikov's for- 


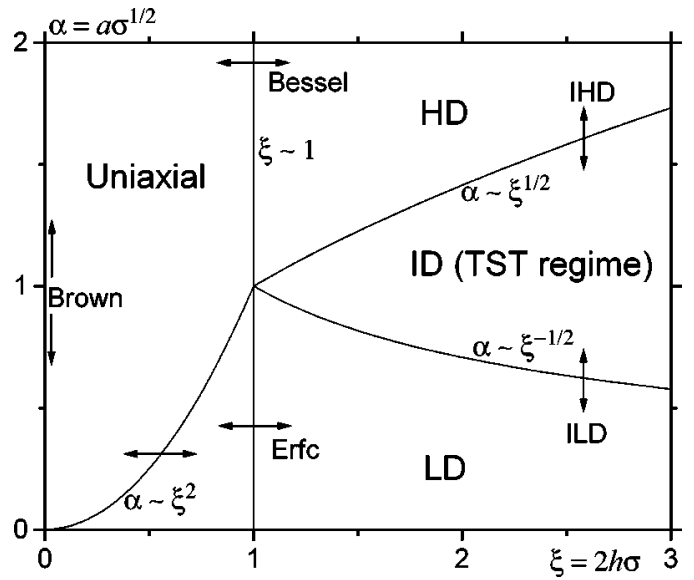

FIG. 2. Different regimes for the escape rate of the uniaxial spin system in the transverse field $(\sigma \gg 1, h \ll 1)$.

mula [12]. The HD-Uniaxial crossover, which is described by Eq. (20), occurs for $\alpha \gg 1$ at $\xi \sim 1$. For $\alpha \ll 1$, there are two crossovers between the LD and uniaxial regimes. One of them occurs at $\xi \sim 1$ and is described by Eqs. (23) and (25). Another one occurs at $\alpha \sim \xi^{2}$.

The latter follows from the perturbative solution for the escape rate at small transverse fields for arbitrary values of $a$. To second order in $h$, the result has the form

$$
\Gamma(h) / \Gamma(0) \cong 1+\left(\xi^{2} / 4\right) F(\alpha)=1+h^{2} \sigma^{2} F(\alpha),
$$

where

$$
\begin{aligned}
F(\alpha) & =1+\frac{1}{\alpha^{2}} \int_{0}^{1} d u \exp \left[\frac{\ln (1-u)+u}{2 \alpha^{2}}\right] \\
& =1+2\left(2 \alpha^{2} e\right)^{1 /\left(2 \alpha^{2}\right)} \gamma\left(1+1 /\left(2 \alpha^{2}\right), 1 /\left(2 \alpha^{2}\right)\right),
\end{aligned}
$$

and $\gamma(a, z)=\int_{0}^{z} d t t^{a-1} e^{-t}$ is an incomplete gamma function. The limiting forms of the above expression are

$$
F \cong \begin{cases}1+1 / \alpha-1 /(2 \alpha)^{2}+\cdots, & \alpha \gg 1 \\ \sqrt{\pi} / \alpha-1 / 3+\sqrt{\pi} \alpha / 6+\cdots, & \alpha \ll 1 .\end{cases}
$$

The last formula shows that for $\alpha \ll 1$ the escape rate $\Gamma(h)$ essentially deviates from its uniaxial value if $\alpha \sim \xi^{2}$. This

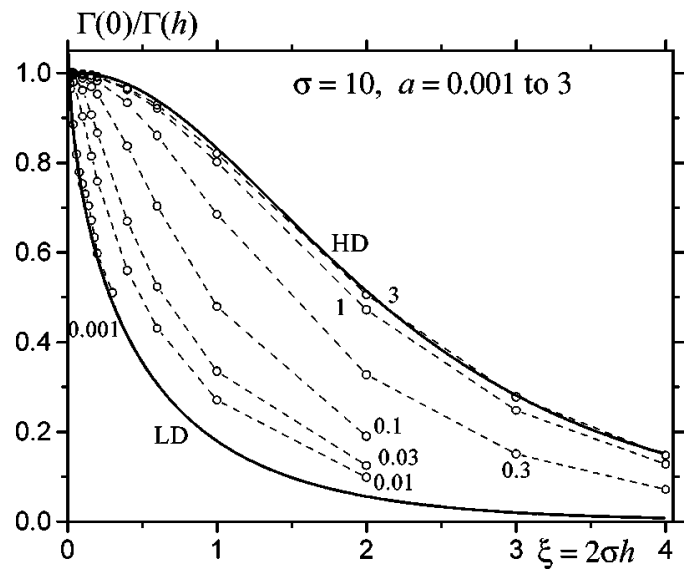

FIG. 3. Transverse-field dependence of the inverse relaxation rate for $\sigma=10$ and different values of the damping constant $a$. Solid lines represent Eq. (11) with $A$ given by Eqs. (20) and (23) with $Q=2^{3 / 2} / \pi \approx 0.900$.

defines the other crossover mentioned above.

The results of the numerical calculation of the thermal activation rate as the lowest eigenvalue of the Fokker-Planck equation [9] are shown in Fig. 3. For large $a$ the agreement with the HD-Uniaxial crossover formula, Eq. (20), is rather good. For small $a$ the field interval is restricted due to convergence problems. Nevertheless, there good accord with the LD crossover formula, Eq. (23), in the region $\xi \lesssim 1$, where the standard LD result of Eq. (19) is invalid.

We have shown how different damping-dependent regimes of thermal activation for uniaxial magnetic particles with transverse field merge into the single uniaxial regime when the field tends to zero, and presented the complete "phase diagram" of the different regimes. The uniaxial characteristics appear for $\xi \lesssim 1$, i.e., for $h \lesssim 1 / \sigma$ [see Eq. (9)]. It should be noted also that the transition from classical to quantum regimes of the escape with decreasing temperature is strongly modified by proceeding to the uniaxial limit [13]. The latter is, however, a more pronounced effect and it already occurs for $h \leqslant 1 / 4$ for our model.

W.T.C. thanks the Enterprise Ireland Research Collaboration fund for the support for this work. D.S.F.C. and E.K. thank UKEPSRC Grant Nos. JR/L06225 and JR/L21891.
[1] L. Néel, Cah. Phys. 25, 1 (1944).

[2] W. F. Brown, Jr., Phys. Rev. 130, 1677 (1963).

[3] W. F. Brown, Jr., IEEE Trans. Magn. MAG-15, 1196 (1979).

[4] W. Wernsdorfer, E. Bonet Orozco, K. Hasselbach, A. Benoit, B. Barbara, N. Demoncy, A. Loiseau, and D. Mailly, Phys. Rev. Lett. 78, 1791 (1997).

[5] E. C. Stoner and E. P. Wohlfarth, Philos. Trans. R. Soc. London, Ser. A 240, 599 (1948).

[6] W. T. Coffey, D. S. F. Crothers, J. L. Dormann, Yu. P. Kalmykov, E. C. Kennedy, and W. Wernsdorfer, Phys. Rev. Lett. 80, 5655 (1998).
[7] W. T. Coffey, D. S. F. Crothers, J. L. Dormann, L. J. Geoghegan, E. C. Kennedy, and W. Wernsdorfer, J. Phys.: Condens. Matter 10, 9093 (1998).

[8] L. J. Geoghegan, W. T. Coffey, and B. Mulligan, Adv. Chem. Phys. 100, 475 (1997).

[9] W. T. Coffey, D. S. F. Crothers, J. L. Dormann, L. J. Geoghegan, and E. C. Kennedy, Phys. Rev. B 58, 3249 (1998).

[10] I. Klik and L. Gunther, J. Stat. Phys. 60, 473 (1990).

[11] H. A. Kramers, Physica (Amsterdam) 7, 284 (1940).

[12] V. I. Mel'nikov, Physica A 130, 606 (1985).

[13] E. M. Chudnovsky and D. A. Garanin, Phys. Rev. Lett. 79, 4469 (1997). 